\title{
Ophthalmic Diagnostic Imaging: Retina
}

\author{
Philipp L. Müller, Sebastian Wolf, Rosa Dolz-Marco, \\ Ali Tafreshi, Steffen Schmitz-Valckenberg, \\ and Frank G. Holz
}

\subsection{Introduction}

In the past decades, optical coherence tomography (OCT) has been established as one of the most important imaging modalities in clinical practice for the diagnosis and follow-up of patients with retinal diseases, as well as a source for outcome measurements in clinical trials. Using backscattered light waves from the retina that interfere with a reference beam, it enables an in-vivo depth profile of the tissue. Modern improvements of this interferometry technique achieve non-invasive visualization of chorioretinal structures close to histology with an axial resolution of under $7 \mu \mathrm{m}$ (Fig. 4.1) [1, 2].

\section{P. L. Müller}

Department of Ophthalmology, University of Bonn, Bonn, Germany

Moorfields Eye Hospital, NHS Foundation Trust, London, UK

S. Wolf

Department of Ophthalmology, University of Berne, Berne, Switzerland

R. Dolz-Marco

Heidelberg Engineering, Heidelberg, Germany

Unit of Macula, Oftalvist Clinic, Valencia, Spain

A. Tafreshi $(\bowtie)$

Heidelberg Engineering, Heidelberg, Germany

S. Schmitz-Valckenberg · F. G. Holz

Department of Ophthalmology, University of Bonn, Bonn, Germany
The first commercially available OCT devices were based on time-domain detection that featured rather low scan rates of $400 \mathrm{~A}$-scans per second leading to possible errors associated with eye motion and reduced measurement accuracy as well as reproducibility (Fig. 4.1a). Nevertheless, it became widely accepted for the assessment of various retinal diseases [3, 4]. Subsequently, the spectral domain (SD) and swept source (SS) imaging technologies have dramatically improved sampling speed and signal-to-noise ratio by using a highspeed spectrometer that measures light interferences from all time delays simultaneously or a tunable frequency swept laser light source (that sequentially emits various frequencies in time) and photodetectors instead of a spectrometer to measure the interference, respectively [5].

For SD-OCT devices, technical improvements has enabled scan rates up to $250,000 \mathrm{~Hz}$ in commercially available devices $[6,7]$. The Spectralis ${ }^{\circledR}$ device by Heidelberg Engineering (Heidelberg, Germany) was the first commercially available SD-OCT device that combines the OCT technique with a confocal scanning laser ophthalmoscope (cSLO) using a near-infrared laser light source (815 nm, Fig. 4.1b). The cSLO features simultaneous eye-tracking based on a retinal fundus reflectivity image, enabling accurate and repeatable alignment of OCT images, advanced noise reduction and an auto rescan function for precise placement of follow-up scans [8].

Commercial SS-OCT devices employ a longer wavelength $(>1050 \mathrm{~nm})$ laser light source and 

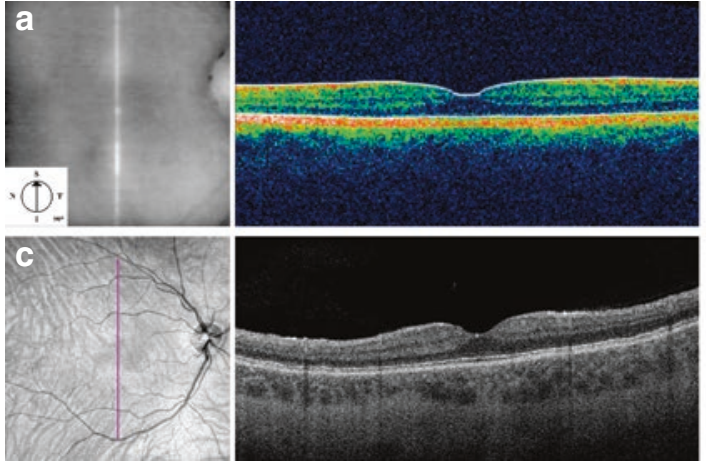

Fig. 4.1 Evolution of OCT imaging in retinal diagnostics. The different generations of OCT imaging devices in exemplary healthy subjects is demonstrated. (a) The time-domain OCT (Stratus OCT, Carl Zeiss Meditec, Jena, Germany) enables the investigator to get a 3-dimensional impression of the retina and retinal layers despite restricted axial and lateral resolutions. Therefore, retinal pathologies can more easily be localized and followed up. (b) The spectral-domain OCT (Spectralis ${ }^{\circledR}$, Heidelberg Engineering, Heidelberg, Germany) combines the OCT technique with a confocal scanning laser ophthalmoscope for eye tracking (left) and distinctly improves resolution and sampling speed, allowing for seg-

have scan rates as fast as $200,000 \mathrm{~Hz}$. The longer wavelengths is thought to enhance visualization of subretinal tissue and choroidal structures (Fig. 4.1c) [9-13]. Similar effects are aspired by techniques like image averaging and/or enhanced depth imaging (Fig. 4.1d).

The astounding clinical implications and the numerous potential research applications have led to the rapid acceptance and integration of OCT and cSLO technology in the ophthalmic community. Ongoing improvements of the technologies will further deepen the understanding of the physiology and pathophysiology of various retinal conditions as a prerequisite for-the development and approval of new therapeutic approaches. This chapter aims to review the role of OCT diagnostics in retinal conditions, with particular emphasis on differential diagnoses as well as monitoring of progression and therapeutic outcomes.

\subsection{Application of OCT in Retinal Diagnostics}

OCT technology has revolutionized modern ophthalmology during the last decades. By now, OCT is widely used in clinical practice and trials,

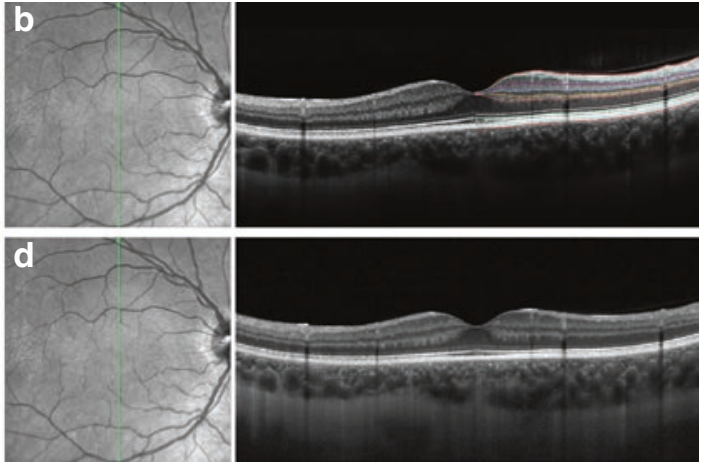

mentation of individual retinal layers (colored lines at the right). These improvements have made OCT one of the most important diagnostic devices for differential diagnosis, determination of progression or treatment effects, and treatment indication in clinical routine as well as in study environments. However, due to imaging wavelength, visualization of deeper structures (i.e. choroid) may be limited. Other OCT imaging techniques like (c) the swept-source OCT (PLEX Elite 9000, Carl Zeiss Meditec, Dublin/CA, USA) or (d) the enhanced depth imaging mode enhance the visualization of subretinal tissue while detail of superficial retinal layers is reduced

as it is a noninvasive, quick and reproducible imaging modality. Advancements in OCT technology have improved the differential diagnosis, the knowledge of the physiopathology, and the ability to monitor disease progression as well as therapeutic effects. Diagnostic capabilities will be reviewed across a range of retinal conditions, including common diseases such as age-related macular degeneration (AMD), diabetic retinopathy, retinal vascular diseases, and rare retinal diseases including hereditary dystrophies. The depth resolution of individual retinal layers allows for localization of altered structures, enabling differentiation of diseases affecting the outer retina from pathologies that primarily impact on the inner retina. The precision and accuracy of the technology further allow for visualization and clinical assessment of subtle structural alterations or different disease stages.

\subsubsection{Age-Related Macular Degeneration}

In the developed world, AMD is the leading cause of irreversible visual impairment in adults with an age over 60 years [14]. OCT imaging 
allows for a 3-dimensional visualization and assessment of the integrity or disruption of each individual retinal layer, providing a precise detection of early changes, both in the atrophic and the neovascular spectrum of the disease [14].

The clinical hallmark of AMD is the deposition of acellular, polymorphous material between the retinal pigment epithelium (RPE) and Bruch's membrane ('drusen') as well as the appearance of pigmentary changes (hyper- and hypopigmentation) [15]. AMD-related drusen can be differentiated into soft drusen and cuticular drusen by combining OCT and cSLO imaging characteristics. Other deposits located above the RPEBruch's membrane band correspond with reticular pseudodrusen (Fig. 4.2) and acquired vitelliform lesions [16]. Soft drusen are represented by discrete areas of RPE elevation with variable reflectivity, reflecting the heterogenic composition of the underlying material (Fig. 4.2a) [17, 18]. Large confluent drusen may sometimes be accompanied by fluid accumulation under the retina that is seen in the depression between drusen. Ruling out the presence of choroidal neovascularization is important in order to avoid unnecessary treatment with anti-angiogenic therapies [19], and OCTangiography (described in Chap. 6) images may be useful in these challenging cases. Drusen can further be accompanied by discrete changes in the overlying neurosensory retina including disruption of the ellipsoid zone band, the external limiting membrane, thinning of the outer nuclear layer or intraretinal pigment clumping and migration that can be visualized by OCT $[18,20]$.

Cuticular drusen were first described as 'basal laminar drusen' by Gass in 1974 as numerous, small, round, uniformly sized, yellow, sub-RPE lesions that show early hyperfluorescence on fluorescein angiography resulting in a "starry night" appearance [21, 22]. The ultrastructural and histopathological characteristics of cuticular drusen are similar to those of hard drusen, however, their lifecycle and macular complications are more comparable with those of soft drusen [23]. On OCT, cuticular drusen are classically described as a saw-tooth elevation of the RPE with rippling (and occasional disruption) of the overlying ellipsoid zone band and the external limiting membrane (Fig. 4.2b) [24].
Reticular pseudodrusen were first described in 1990 as a peculiar yellowish pattern in the fundus of AMD patients, and in 1991 as an ill-defined network of broad interlacing ribbons [25, 26]. OCT enabled an improved characterization of reticular pseudodrusen (Fig. 4.2c) showing that these lesions correspond to granular hyperreflective material between the RPE and the ellipsoid zone band. As a result, the term 'subretinal drusenoid deposits' has been proposed [27].

Drusen may be accompanied by acquired vitelliform lesions that are believed to occur as a result of RPE dysfunction leading to impaired photoreceptor outer segment turnover. Acquired vitelliform lesions are clinically apparent as yellowish material and mimic the appearance of choroidal neovascularisation $(\mathrm{CNV})$ on fluorescein angiography. In OCT imaging, the subretinal heterogeneous material is well separable from fluid [28]. In some cases, the RPE phagocytoses the subretinal material leading to either a resolution of the lesion or an atrophy of the RPE and the outer retinal layers. However in other cases, a conversion into a neovascular form is seen (Fig. 4.3) [27, 28].

It has been shown that drusen diameter and volume are a significant risk factor for progression to advanced AMD. Therefore, early and intermediate AMD is differentiated inter alia by smaller and larger than $125 \mu \mathrm{m}$ drusen size, respectively [16]. As manual analysis of drusen on color fundus images is not reliable and practical, efforts are underway to use OCT for automated detection and quantification of drusen size, area, and volume. This may help to identify patients at high risk of disease progression and to institute appropriate upcoming prophylactic interventions [27].

Late AMD forms include macular atrophy and neovascular AMD. Macular atrophy is defined by areas of RPE atrophy that are accompanied with loss of photoreceptors and varying degrees of choroidal impairment, in the absence of neovascularization, the term geographic atrophy (GA) is frequently used [29]. On OCT, GA appears as areas of sharply demarcated choroidal hyperreflectivity from loss of the overlying RPE associated with thinning or loss of the outer retinal layers and eventually choroidal thinning that 
a

\section{Soft drusen}
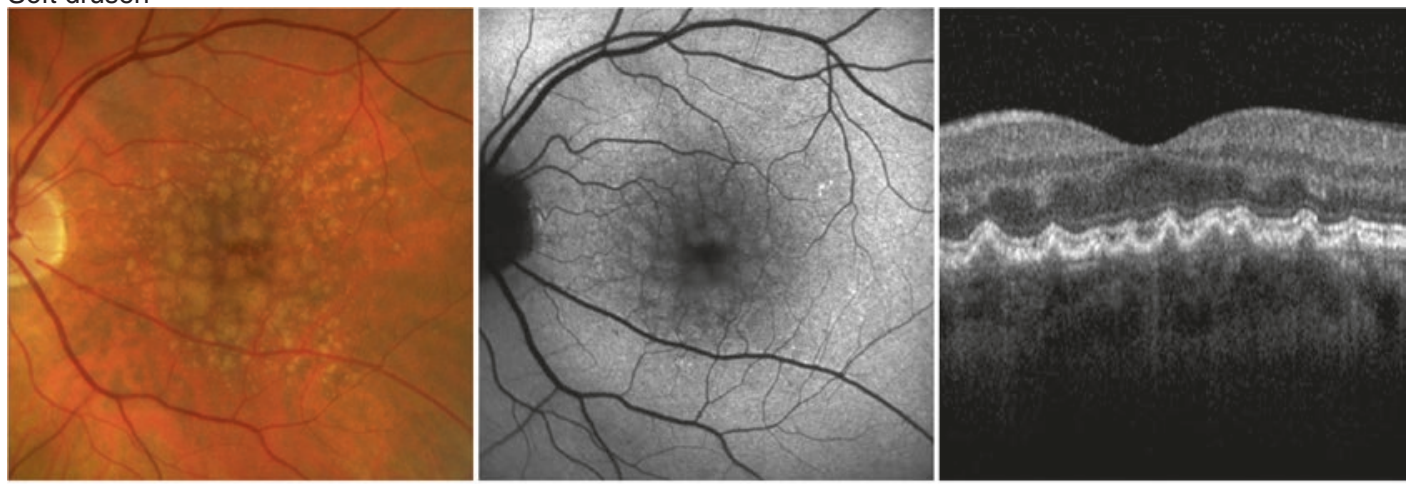

b

\section{Culticular}
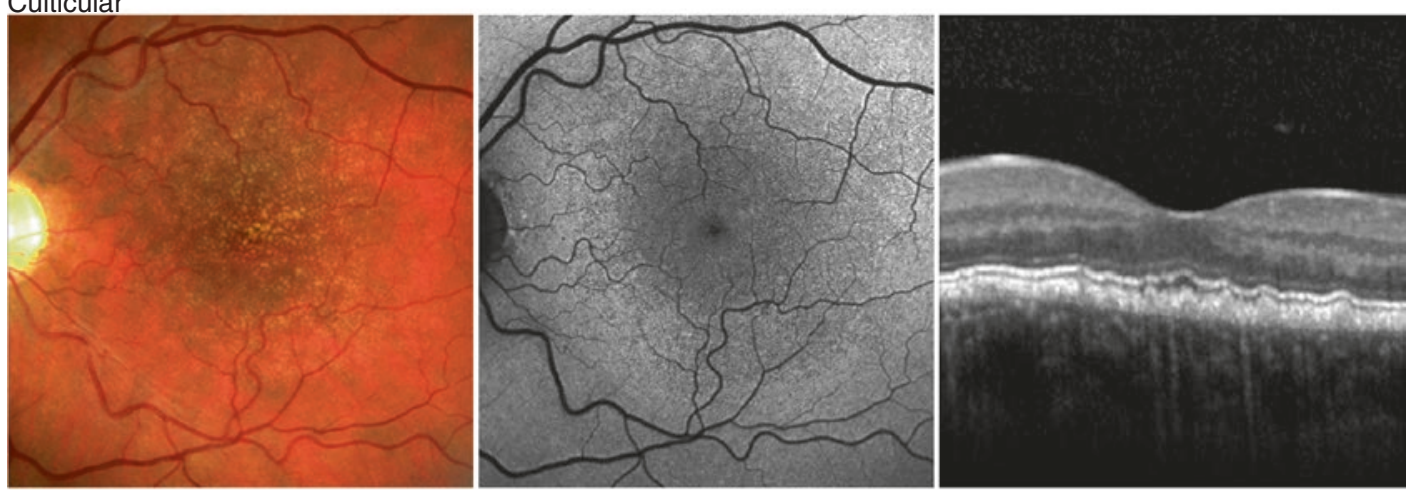

C

\section{Reticular Pseudodrusen}
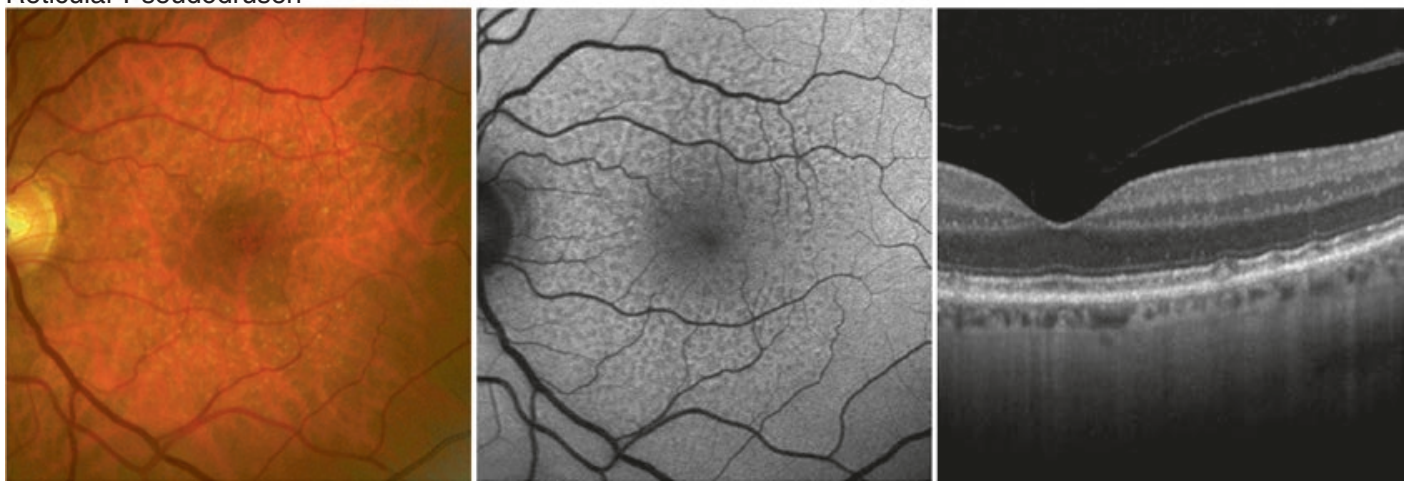

Fig. 4.2 (a-c) Subtypes of AMD related drusen. From left to right, fundus color, fundus autofluorescence, and optical coherence tomography images of soft drusen, cuticular drusen and reticular pseudodrusen are shown. Source: Gliem
M et al:: Quantitative Fundus Autofluorescence in Early and Intermediate Age-Related Macular Degeneration. JAMA Ophthalmology. 2016. Reprinted with permission. This figure is not covered by the CC BY license can be tracked over time with this technique [30, 31]. As OCT imaging is not affected by macular pigment, the reproducibility of GA progression measurements, especially in patients with foveal sparing disease manifestation, is preserved (Fig. 4.4) [27]. Furthermore, OCT enables the imaging of subtle changes as regressing drusenoid material, islands of preserved photoreceptors within GA or in the junctional zone, and even preapoptotic stage of neuronal cellular elements can be clearly visualized [32]. Evaluation of choroidal alterations and junctional zones of GA 

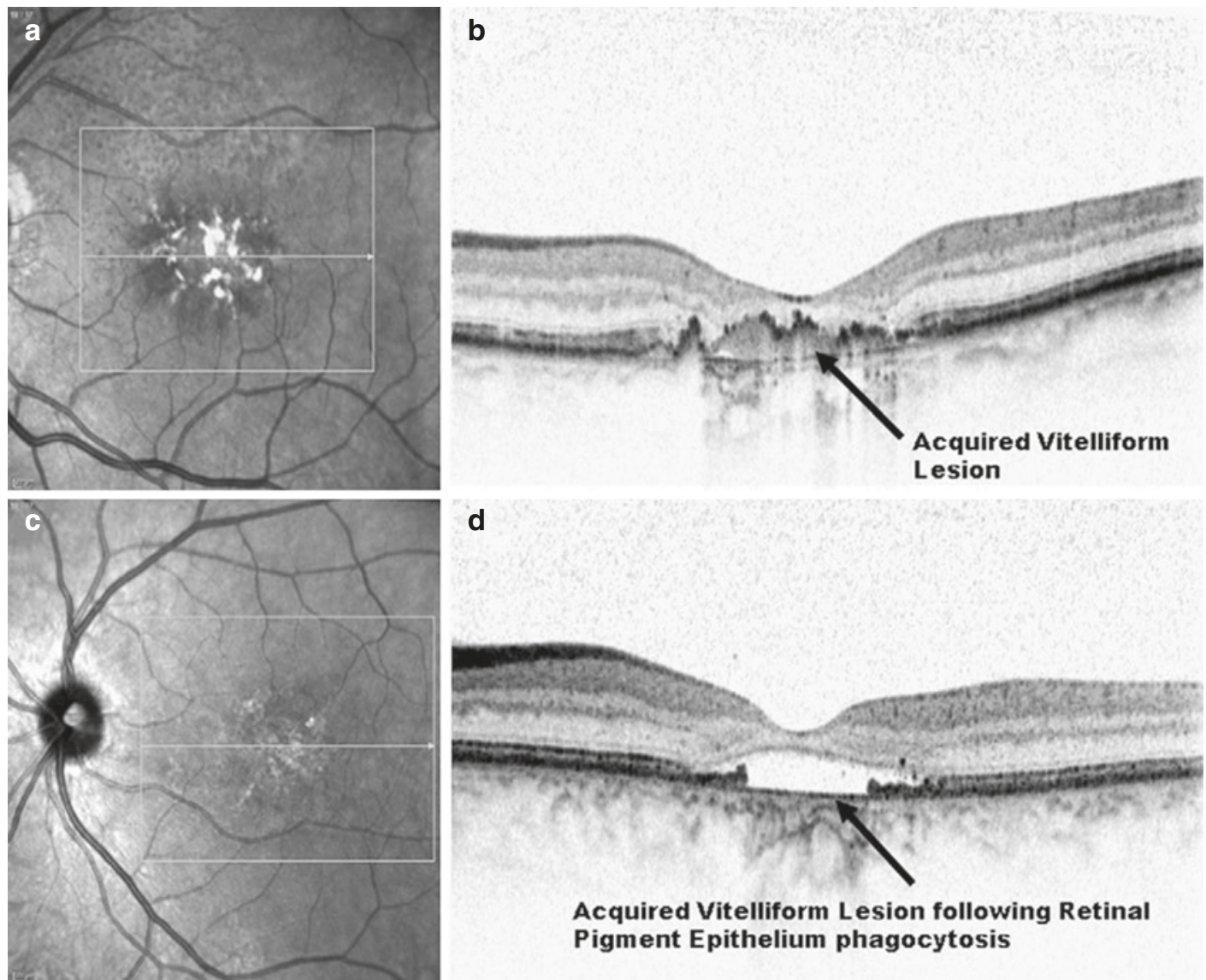

Acquired Vitelliform Lesion following Retinal Pigment Epithelium phagocytosis

Fig. 4.3 Acquired vitelliform lesions. Acquired vitelliform lesions are localized to the subretinal space $(\mathbf{a}, \mathbf{b})$. in progression, the subretinal material may be phagocytosed and the acquired vitelliform lesions may seem to contain subretinal

on OCT and cSLO images further provide insight into the pathogenesis of GA and the relative roles of choriocapillaris, RPE and photoreceptors in the initiation and propagation of this condition. This allows for definition of future treatment targets as well as estimation of individual progression speed [33-35].

In neovascular AMD, abnormal blood vessels develop from the choroidal circulation (choroidal neovascularization) or, from the retinal circulation (retinal angiomatous proliferation, RAP) $[36,37]$. Based on a histological and OCT classification, anatomical classification was proposed coining the terms type 1 , type 2 and type 3 neovascularization $(\mathrm{NV})$. Type $1 \mathrm{NV}$ is located between the RPE band and Bruch's membrane, fluid on OCT (c, d). Source: Keane P et al.: Evaluation of Age-related Macular Degeneration With Optical Coherence Tomography. Survey of Ophthalmology. 2012. Reprinted with permission. This figure is not covered by the CC BY license

Type $2 \mathrm{NV}$ is located above the RPE band in the subretinal space, and Type $3 \mathrm{NV}$ is originated from the deep capillary plexus of the retina and located in the outer retinal layers. The proliferation of the immature vessels results in fluid exudation and hemorrhage, leading to the formation of cystoid lacunae between the RPE and Bruch's membrane (retinal pigment epithelial detachment, PED), between the neurosensory retina and the RPE (serous retinal detachment), and within the retinal extracellular space (intraretinal fluid; Fig. 4.5a) [27]. The associated invasion of fibroblasts result in disciform scar formation with loss of the RPE and overlying photoreceptors and significant disorganization of the overlying retinal architecture [38]. By using OCT, each of these 

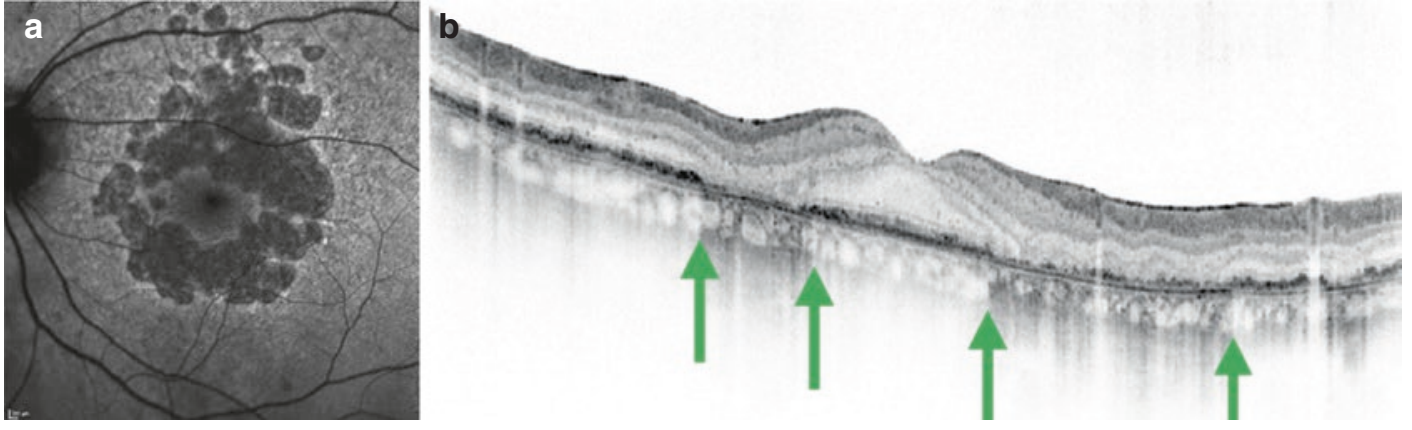

Fig. 4.4 Geographic Atrophy. Foveal sparing geographic atrophy is demonstrated by (a) fundus autofluorescence imaging (excitation wavelength, $488 \mathrm{~nm}$ ) and (b) OCT. Due to shadowing of macular pigment, the affection of the fovea in fundus autofluorescence images may be difficult to determine. In OCT images, area of geographic atrophy is well demarcated due to choroidal hyperreflectivity. Source: Lindner M et al.: Directional Kinetics of Geographic Atrophy Progression in Age-Related Macular Degeneration with Foveal Sparing. Ophthalmology. 2015. Reprinted with permission. This figure is not covered by the CC BY license
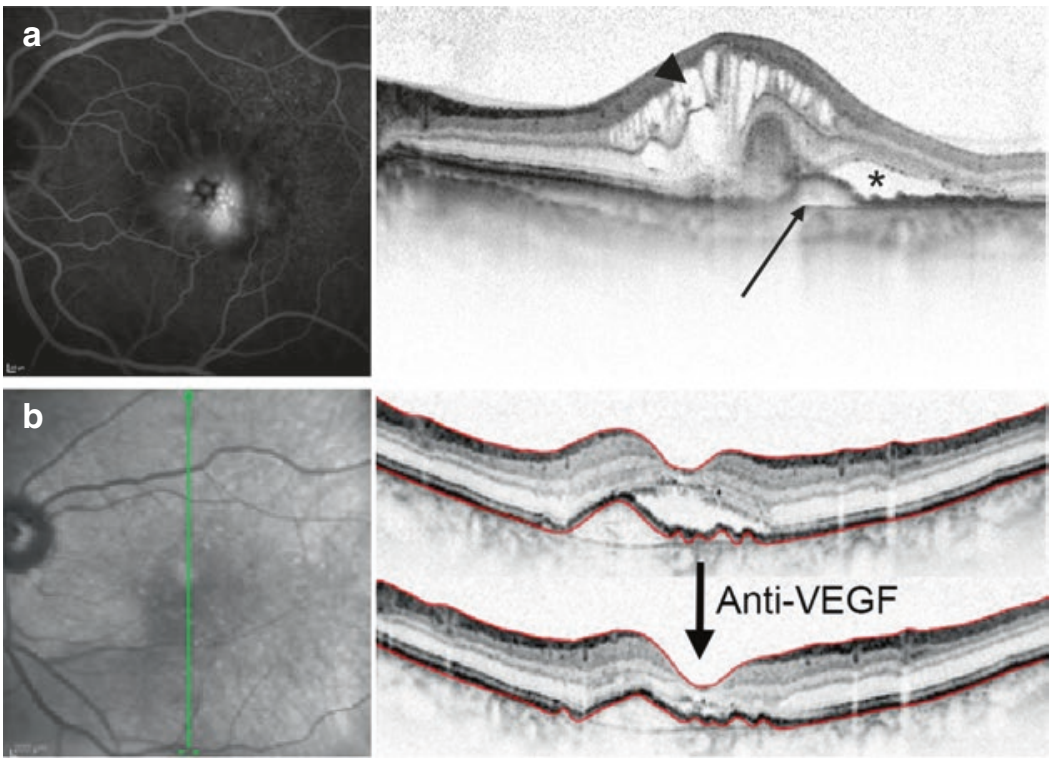

Fig. 4.5 Neovascular AMD. In OCT imaging of neovascular AMD, pigment epithelium detachments (arrow) appear as elevations of the RPE band relative to Bruch's membrane, subretinal (asterix) and intraretinal (arrowhead) fluid as transparent lacunae associated with leakage in fluorescence angiography (left, a). As treatment effect of antiangiogenic therapy is highly visible in 3-dimensional OCT images, OCT has become the gold standard for therapy monitoring (b) disease-associated changes can be visualized in a 3-dimensional manner. Therefore, treatment indications as well as anti-angiogenic treatment effects can be evaluated much more objectively and precisely than with summation images as provided by invasive fluorescence angiography alone, making the combination of OCT and fluorescence angiography the gold standard imaging strategy for diagnosing neovascular AMD (Fig. 4.5b) [39]. Other diseases associated with clinical macular edema, including central serous chorioretinopathy (CSCR) and polypoidal choroidal vasculopathy (PCV), can further be differentiated more easily from neovascular AMD as they differ in OCT appearance (e.g., thicker choroid) [40]. This might be of specific impor- 
tance in retinal diseases that are not responding to antiangiogenic treatment (see following subchapters).

\subsubsection{Diabetic Retinopathy and Macular Edema}

Worldwide, diabetic retinopathy is the leading cause of visual impairment in the working-age population. Similar to AMD, diabetic retinopathy is assessed by a multimodal approach, especially as the pathogenesis and clinical features are primarily attributed to retinal vascular damage. Thus, fluorescein angiography plays a key role in the diagnosis of the disease. Recent OCT findings indicate that choroidal angiopathy may also be involved, providing further insight into the pathogenesis of diabetic retinopathy. Choroidal thinning is present in patients with diabetic retinopathy and related to disease severity (Fig. 4.6). Therefore, choroidal thickness analysis using OCT may be an important parameter to assess the severity of diabetic retinopathy [41-43].

As macular edema is one of the major complications of diabetic retinopathy, well treatable with laser treatment, anti-angiogenic, steroid therapy or a combination of those, a reliable diagnostic and treatment monitoring module is needed [44]. The combination of OCT imaging and fluorescence angiography has become the gold standard imaging strategy in diabetic macular edema, providing high-resolution 3-dimensional retinal information [45-47].

\subsubsection{Retinal Vascular Occlusions and Other Vascular Conditions}

In retinal vascular disease, it is undisputed that fluorescence angiography is the diagnostic gold standard. However, macular edema caused by excessive VEFG production may occur. In these cases, laser treatment, intravitreal dexamethasone or antiangiogenic injections have been shown to stabilize and even improve the anatomy as well as the visual acuity of these patients [48].
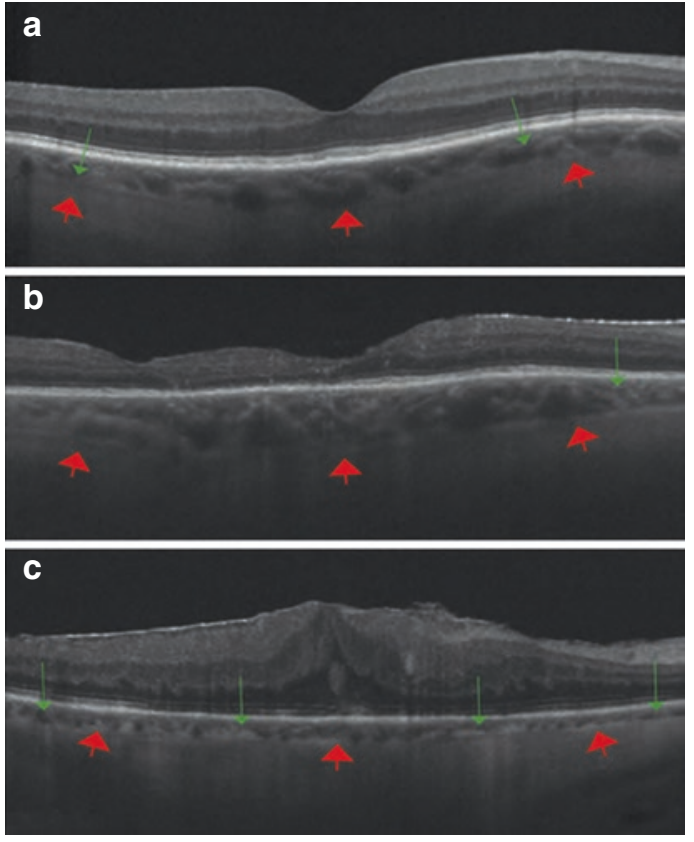

Fig. 4.6 OCT features of diabetic retinopathy. The OCT images of nonproliferative diabetic retinopathy (a), proliferative diabetic retinopathy (b), and diabetic macular edema (c) revealed thinner choroid. Of note, the latter reveald most diffuse choroidal thinning. Proliferative diabetic retinopathy showed paracentral loss of mainly inner retinal structures. Red arrows highlight the choroid-sclera interface. Focal thinning is indicated by green arrows. Source: Adhi M \& Dunker J: Optical coherence tomography--current and future applications. Current opinion in ophthalmology. 2013. Reprinted with permission. This figure is not covered by the CC BY license

For treatment monitoring as well as evaluation of prognosis, OCT is of great value as it provides 3D structural information concerning the involved area and the severity (Fig. 4.7). In eyes with macular edema secondary to retinal vein occlusions, OCT images may show the presence of hyporeflective spaces within the retinal nerve fiber layer that can predict the presence of retinal non-perfused areas, as well as the status of the photoreceptor layer that directly correlates with the visual acuity. In cases showing arterial ischemia, location of retinal hyperreflectivity involving the middle retinal layers may locate the ischemic injury involving the deep capillary plexus as seen in paracentral acute middle maculopathy. 


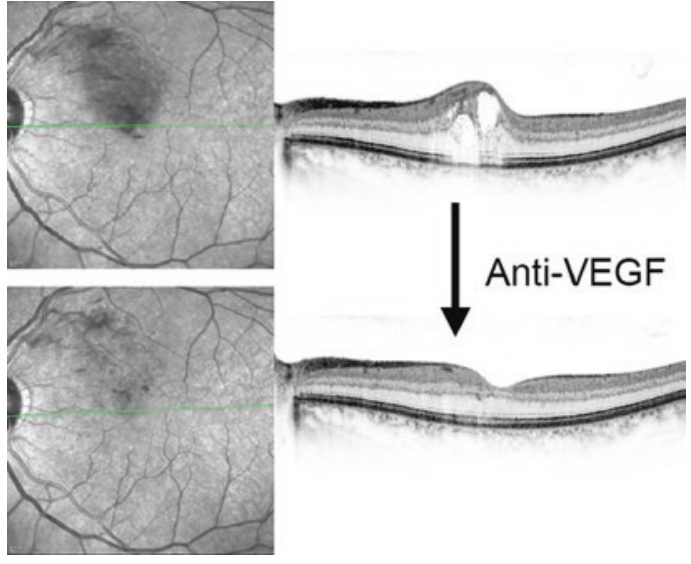

Fig. 4.7 Retinal vein occlusion. Similar to neovascular AMD, treatment effect of steroid and antiangiogenic therapy for macular edema secondary to retinal vein occlusion is highly visible in 3-dimensional OCT images. Therefore, multimodal assessment of OCT and fluorescence angiography is current gold standard for this entities

\subsubsection{Central Serous Chorioretinopathy and Related Diseases}

Central serous chorioretinopathy (CSCR) is typically characterized by a serous retinal detachment in the acute phase, thought to be caused by a generalized disruption of the choroidal vasculature with diffuse hyperpermeability [49]. In OCT imaging, an elevation of the neurosensory retina from the RPE is present, associated with a significant increase in the thickness of the choroid and focal dilation of large choroidal vessels ('pachyvessels') [2]. The latter finding implies the pathophysiologic role of hydrostatic pressure in choroidal vessels and distinguishes CSCR from other causes of subretinal fluid, indicating the need and importance of OCT assessment of choroidal thickness. CSCR usually resolves spontaneously within a few months. However, some patients demonstrate a chronic form with persistent subretinal fluid and eventual permanent visual loss. These cases might further develop secondary $\mathrm{CNV}$ requiring prompt diagnosis to avoid delayed treatment. Even in the absence of $\mathrm{CNV}$, chronic forms of CSCR may require intervention with treatments such as laser photocoagulation and photodynamic therapy (PDT). Recent data showed a significant reduction in choroidal thick- ness following PDT (Fig. 4.8) [50]. Given the widespread use of PDT for the treatment of chronic CSCR, analysis of choroidal thickness by OCT may be a parameter to assess for disease activity following treatment [2].

\subsubsection{Pathologic Myopia}

Eyes with pathologic myopia (refractive error of at least -6 diopters and/or axial length greater than $26.5 \mathrm{~mm}$ ) are at high risk for developing retinal abnormalities. The examination of myopic fundus is challenging due to extreme thinning of retinal and choroidal tissue thus an accurate and complete evaluation may only be performed with high-resolution imaging including OCT. Common findings in pathologic myopia are chorioretinal atrophy (diffuse or patchy), tractional changes (macular holes, epiretinal membranes, retinal schisis, microvascular folds and vascular avulsions). In some cases, the shape of the presents altered known as 'staphylomas'. All these findings can be detected and carefully assessed with OCT scans. NV occurs in $5-11 \%$ of patients with pathologic myopia and is the most common form of exudative disease, within the first four decades of life [51]. OCT in eyes with pathologic myopia is useful to determine the presence of $\mathrm{NV}$ and to monitor the treatment effects. OCT imaging also allows for an accurate differential diagnosis of findings such as subretinal fluid in dome shape maculopathy (Fig. 4.9) [52].

\subsubsection{Inherited Retinal Diseases and Other Macular Conditions}

Among many other inherited disease, Sorsby fundus dystrophy secondary to mutations in TIMP3 (autosomal dominant) and pseudoxanthoma elasticum secondary to mutations in ABCC6 (autosomal-recessive) are frequently associated with NV. In these cases, OCT has become standard procedure for diagnosis, assessment of disease severity, indication for treatment and to determine individual progression rates (Fig. 4.10) [53, 54]. 

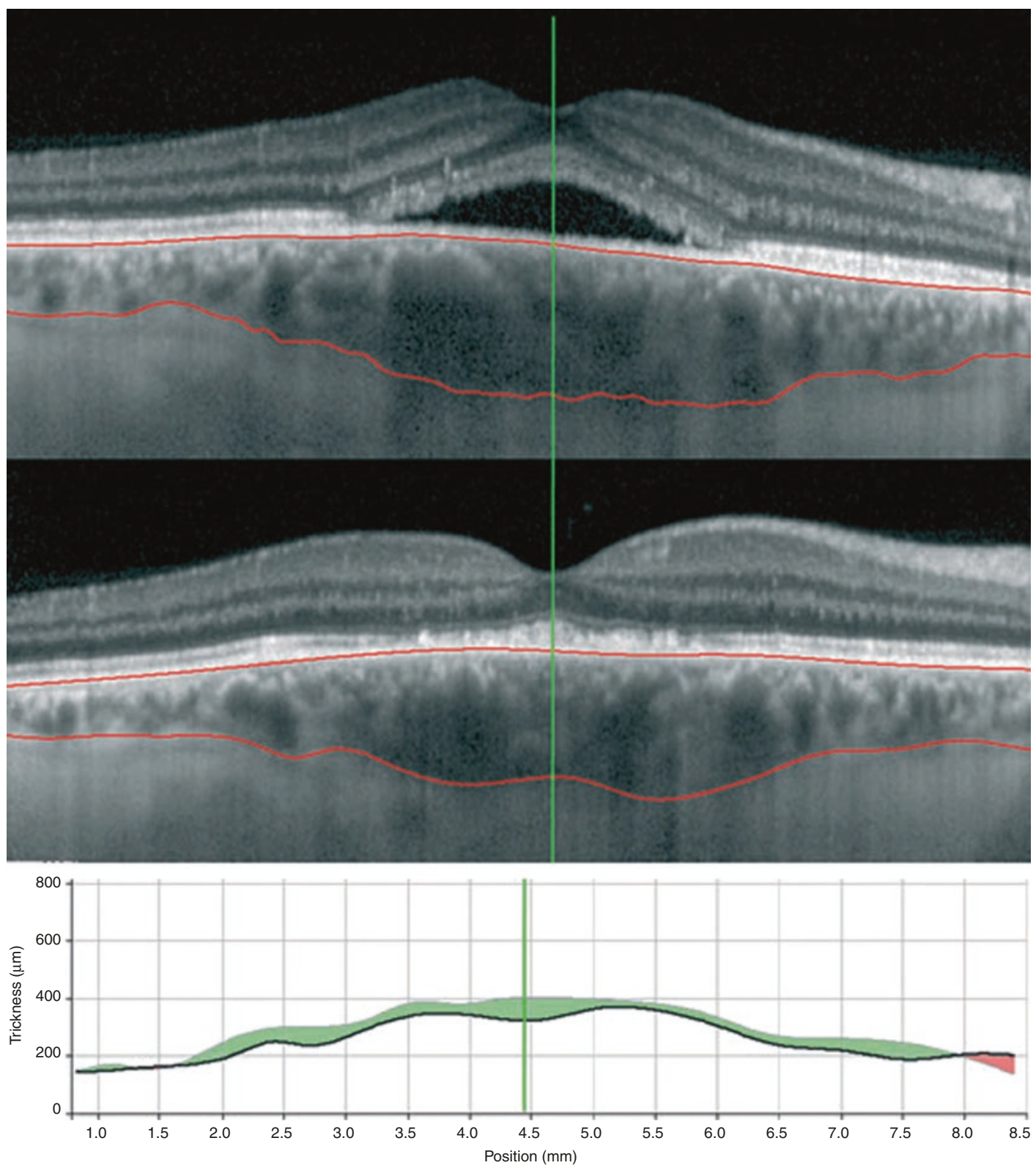

Fig. 4.8 Central serous chorioretinopathy. Horizontal OCT scan from the right eye of a patient with central serous chorioretinopathy before (above) and after (below) verteporfin photodynamic therapy. Treatment was followed by resolution of the subfoveal fluid and by a reduction of the disease associated choroidal thickening, as measured along the red lines indicating the inner and outer borders of the choroid and

Another disease that might be associated with $\mathrm{NV}$ is macular telangiectasia type 2. Using OCT thickness measurements (often in combination with fluorescein angiography), NV lesions are differentiable from degenerative changes that are shown at the bottom as a function of distance. The vertical green line indicates the location of the centre of the fovea. Source: Pryds a \& Larsen M: Choroidal thickness following extrafoveal photodynamic treatment with verteporfin in patients with central serous chorioretinopathy. Acta Ophthalmologica. 2012. Reprinted with permission. This figure is not covered by the CC BY license

regularly seen within the natural progression of this disease [53].

Apart from evaluation of $\mathrm{NV}$ and treatment effects, OCT has a significant value in the assessment and differential diagnosis of inherited 
Fig. 4.9 Myopia. The second most common form of CNV occus secondary to myopia magna. While fluorescence angiography shows only slight leakage at the border of the chorioretinal atrophy (red arrow), associated OCT reveals inhomogeneous material breaking through outer retinal layers with subretinal fluid (green arrow, a). However, subretinal fluid may also be present in myopic eyes with special configuration, called 'dome shape maculopathy' that is often only visible in vertical scans and not responding to antiangiogenic therapy (b)
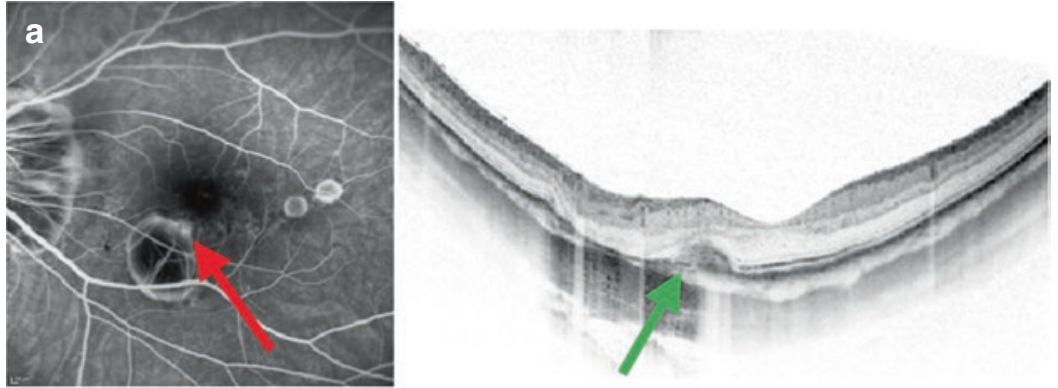

b
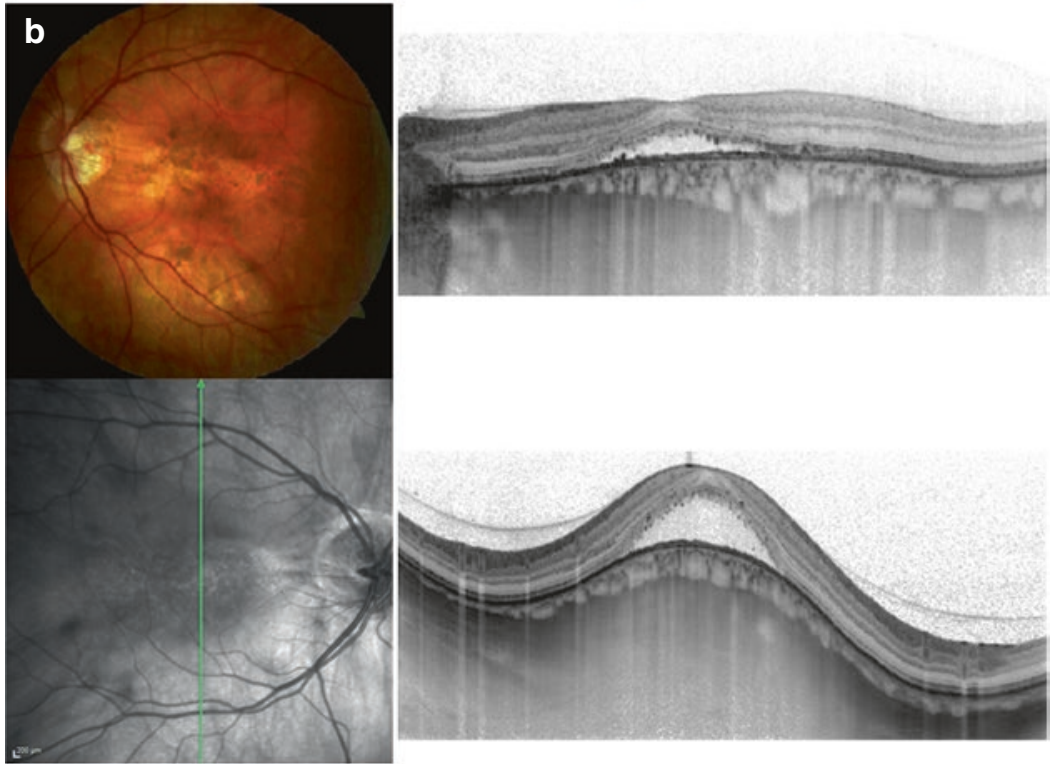

retinal diseases. Recent studies using OCT have provide a new insight regarding the amount of choroidal involvement in the pathogenesis of retinitis pigmentosa, pseudoxanthoma elasticum (PXE) and Stargardt disease [54-56]. The latter even provided evidence for a diffusible factor from the RPE sustaining the choroidal structure.

\subsubsection{Intraocular Tumors}

Pigmented lesions such as choroidal melanomas, nevi or congenital hypertrophy of the RPE and other intraocular tumors such as hemangiomas, hamartomas or osteomas have also been studied using OCT. OCT has enabled improved delineation of tumor borders, with detailed qualitative and quantitative analysis, as well as characterization of reflectivity properties (Fig. 4.11) [57, 58].

\subsubsection{Inflammatory Diseases, Intermediate and Posterior Uveitis}

Intermediate and posterior uveitis may be associated with the development of macular edema, vascular changes in the retina or the choroid, and/or inflammatory lesions. The detection of all these lesions has been enhanced with the use of OCT scans, while providing valuable and reliable information for the challenging follow-up of these patients [59].

\subsubsection{Vitreoretinal Interface}

Detection and detailed evaluation of macular holes, epiretinal membranes and tractional changes have been facilitated by OCT images. The International Vitreomacular Traction Study 

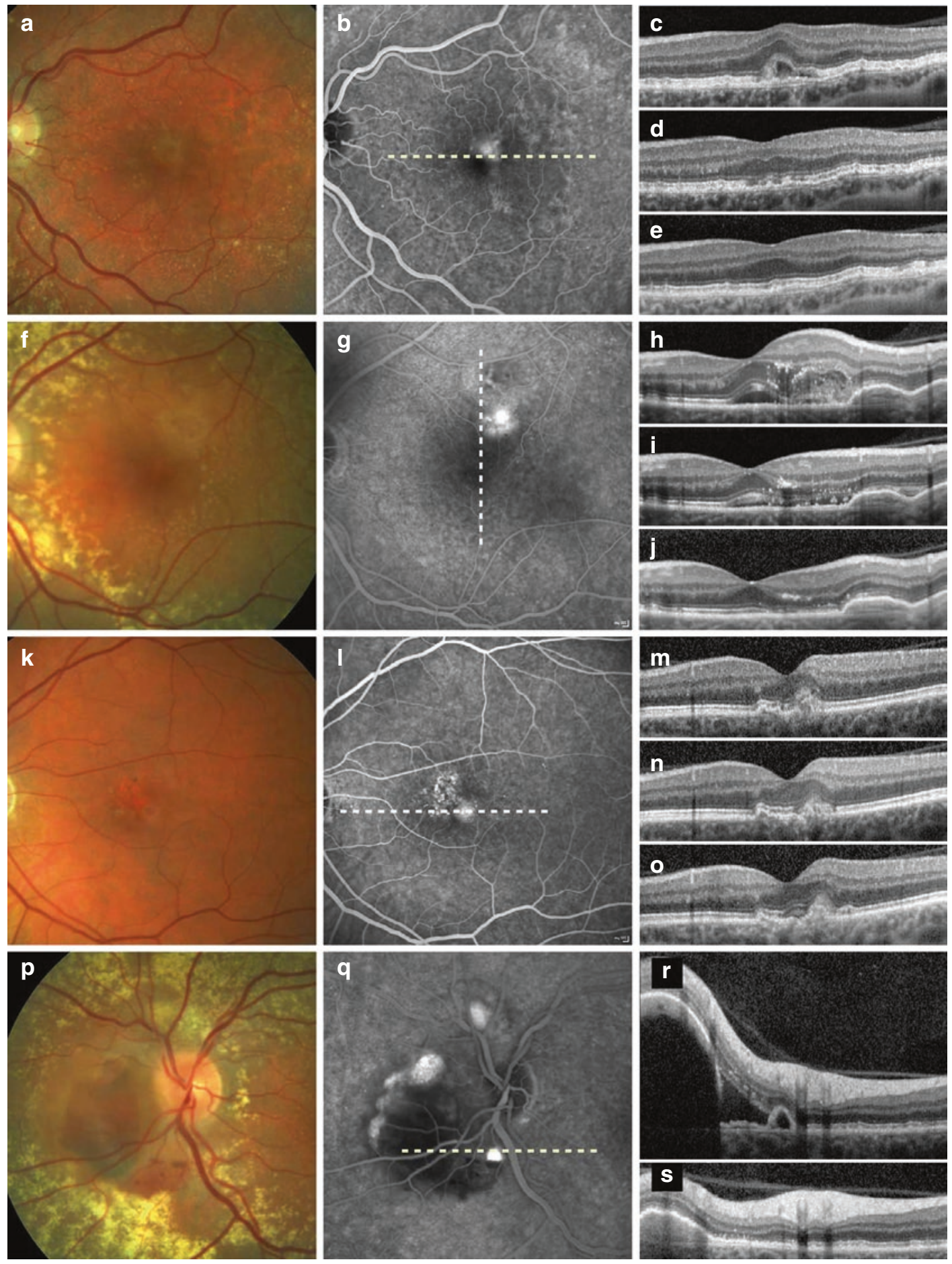

Fig. 4.10 Sorsby Fundus Dystrophy. Color fundus photograph (left), fluorescein angiography (middle), and SD-OCT images (right) demonstrate macular $\mathrm{CNV}(\mathbf{a}-\mathbf{o})$ and juxtapapillary polypoidal choroidal vasculopathy $(\mathbf{p}-\mathbf{s})$ in patients with SFD as well as response to treatment with bevacizumab. All subjects show regression of retinal edema after therapy (lower OCT images). The dotted line marks the position of the respective SD-OCT line scan. Source: Gliem M et al.: Sorsby Fundus Dystrophy: Novel Mutations, Novel Phenotypic Characteristics, and Treatment Outcomes. Invest Ophthalmol Vis Sci. 2015. Reprinted with permission. This figure is not covered by the CC BY license 

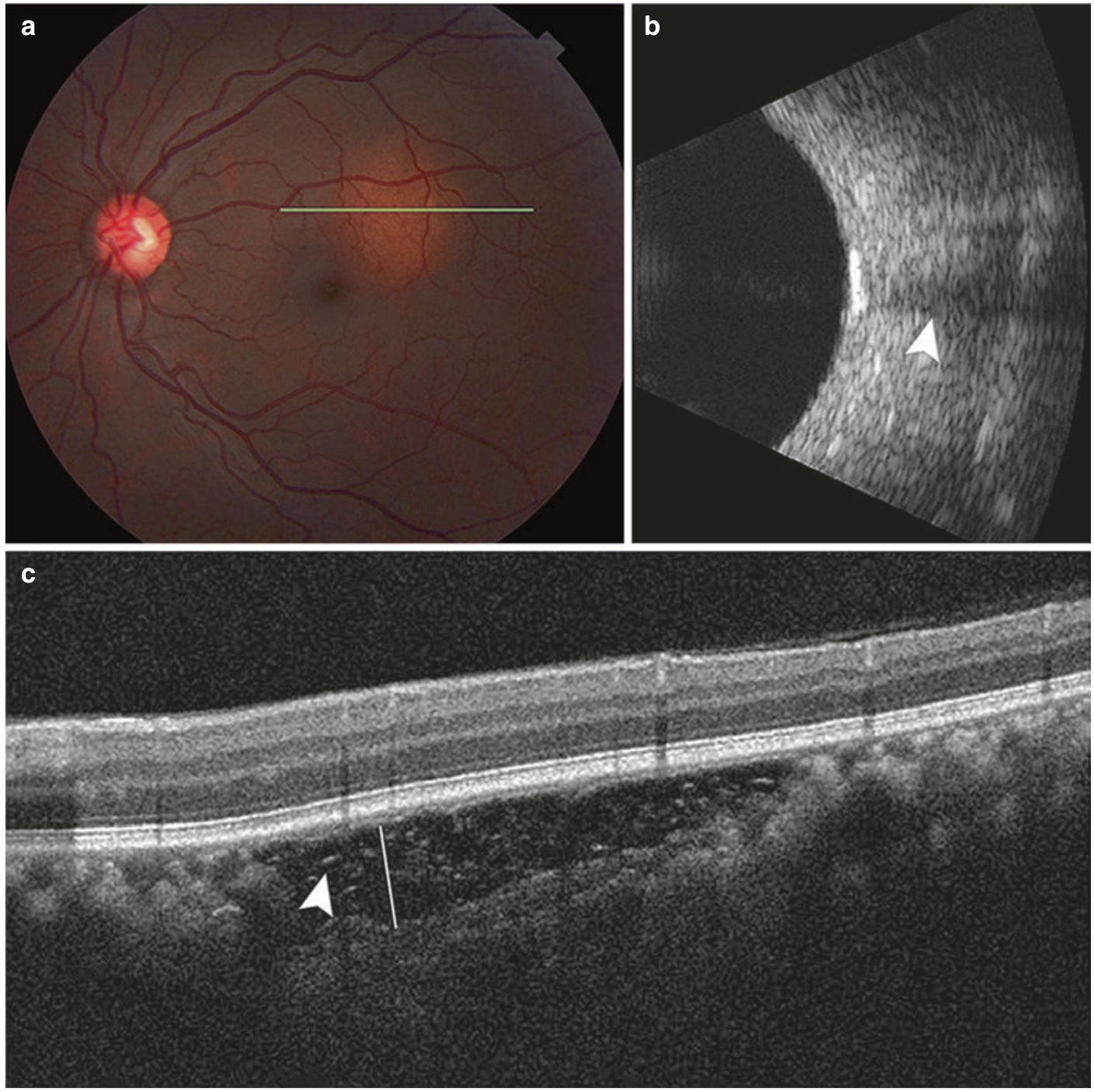

Fig. 4.11 Choroidal osteoma. The color fundus photograph reveals the amelanotic choroidal osteoma in the macula. It measures $3.6 \times 4.1 \mathrm{~mm}$ (a). Ultrasonography reveals a $0.9 \mathrm{~mm}$ thick tumour with posterior shadowing (b, arrowhead). On the OCT-image, the tumour is hyporeflective with intrinsic hyperreflective dots (c, arrowhead). The posterior edge of the tumour is visible

Group classification provided new definitions for vitreomacular adhesion and vitreomacular traction using OCT images [60]. Both can be classified as broad (area of vitreous attachment $>1500 \mu \mathrm{m}$ ) or focal (area of vitreous attachment $\leq 1500 \mu \mathrm{m}$ ). The presence of perifoveal vitreous detachment associated with posterior cortical vitreous attachment within the central $3 \mathrm{~mm}$ may be due to vitreomacular adhesion in the absence of allowing for more accurate tumour thickness measurements. The corresponding (white line) measurement on OCT is $320 \mathrm{~mm}$. Source: Freton A \& Finger PT: Spectral domain-optical coherence tomography analysis of choroidal osteoma. Br J Ophthalmol. 2011. Reprinted with permission. This figure is not covered by the CC BY license

retinal abnormalities, or vitreomacular traction when associated with intraretinal cysts, subretinal fluid, or flattening of the foveal contour, but in the absence of full-thickness interruption of all retinal layers [60].

Full-thickness macular holes are defects of all retinal layers from the inner limiting membrane (ILM) to the photoreceptors with preservation of the RPE located at the level of the fovea. Macular 
holes are classified as small $(\leq 250 \mu \mathrm{m})$, medium $(250-400 \mu \mathrm{m})$, or large $(>400 \mu \mathrm{m})$ based on the size (minimum hole width). Visual outcomes of these cases are related to the size of the hole. A lamellar hole is a partial defect with preservation of the photoreceptors. Macular pseudoholes present as changes in the foveal contour that mimic a lamellar macular hole, without retinal layer defects [60].

Finally, OCT scans allow for visualization and detection of epiretinal membranes as hyperreflective tissue attached to the inner surface of the retina. The location, extension and the evaluation of the outer retinal layers as well as a better planning of the surgical technique is often facilitated by OCT imaging.

\subsection{Pitfalls of OCT in Retinal Diagnostics}

\subsubsection{Acquisition Protocol}

Recent advances have led to reliable and fast acquisition of OCT images, providing a broad application in both clinical and experimental settings [2]. The varying indications for use of OCT technology has raised questions concerning the location, density and interpretation of the scans. While the flexibility of scanning is important in order to optimize the scan protocols, an increasing amount of data requires exponentially larger storage drives and fast broadband network systems [61].

Using adapted imaging protocols dependent on specific diseases may address these challenges. In exudative macular diseases (e.g., NV), volume scans have the advantage of dividing the central retina into equal proportions in order to identify fluid also outside the foveal center. In other diseases such as vitreoretinal interface disorders, the fovea and the optic nerve head are the most important areas, while more eccentrically located retinal zones are less relevant. Radial scans are useful in these cases, giving a more precise representation of a circumscribed retinal area where the scans intersect (i.e., the fovea), compared to eccentric areas (Fig. 4.12) [62]. In unclear cases, planning a more detailed scan protocol in the area of interest should be typically considered.

\subsubsection{Acquisition Technique}

For acquisition of high-quality OCT images, parameters such as alignment of the camera, focus, detector sensitivity and signal strength are important prerequisites. Automatic registration and matching of OCT images of the same retinal location is an essential tool for monitoring subtle changes over time. The same focus should be kept between different imaging sessions and tilting of the head should be avoided during image acquisition in order to minimize artifacts or inaccuracies [63]. Incorrect settings should be identified before a clinician interprets the results. In order to avoid misinterpretations, operators should be adequately trained and instructed to check the quality and completeness of the data directly after the recordings, as an immediate reacquisition might be possible with the subject still in front of the device [61].

Up to now, no common industry standard has been established for OCT imaging. In addition, device-dependent differences may also occur (e.g. in the appearance of retinal thickness), as OCT B-scans are usually displayed as stretched images in the vertical direction. Accordingly, for better comparability, the same patient should be examined with the same device platform over time. Even by simple software updates, the algorithms and definitions of the automatic segmentation lines may change and, therefore, the comparability of subsequent recordings and their evaluation may be limited [61].

\subsubsection{Interpretation}

For an adequate evaluation of modern OCT imaging, the collected data should be reviewed on the reviewer software instead of using printed scans or PDF files, as it offers the possibility to evaluate all collected B-scans individually. Evaluation of a single OCT-scan may not be sufficient for differential diagnosis or to determine disease activity and treatment effects (Fig. 4.13) [61].

Correct interpretation of OCT findings is a prerequisite for treatment decisions. Precise knowledge of retinal and macular diseases is mandatory, as focusing on the relevant findings may be 

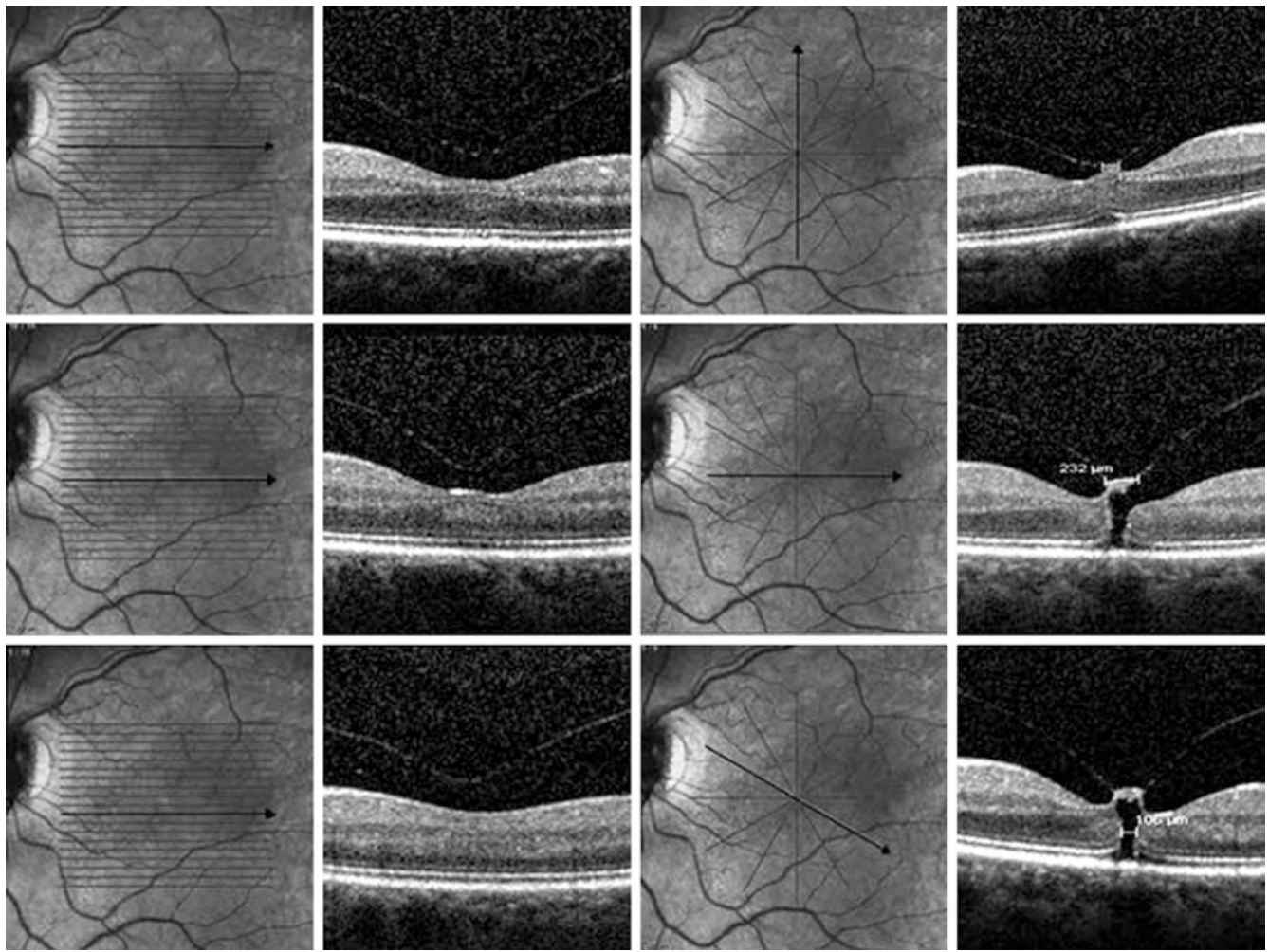

Fig. 4.12 Pitfalls in OCT acquisition. Application of the 19-line volume scan (left panels) versus a star scan protocol (right panels) in an eye with vitreomacular traction and full-thickness macular hole. Note that the three cen-

tral B-scans in the volume scan fail to detect the relevant pathologic findings. Source: Schmitz-Valckenberg S et al.: Pitfalls in retinal OCT imaging. Ophthalmology @ Point of Care. 2017. Reprinted with permission

Fig. 4.13 Pitfalls in OCT interpretation I. Choroidal neovascularization (CNV) under antivascular endothelial growth factor therapy. Evaluation of only the central B-scan for activity of the CNV lesion (a) would fail adequate interpretation of the disease status, because inferior to the fovea, there is intraretinal fluid indicating disease activity (b). Source: Schmitz-Valckenberg S et al.: Pitfalls in retinal OCT imaging.

Ophthalmology@Point of Care. 2017. Reprinted with permission
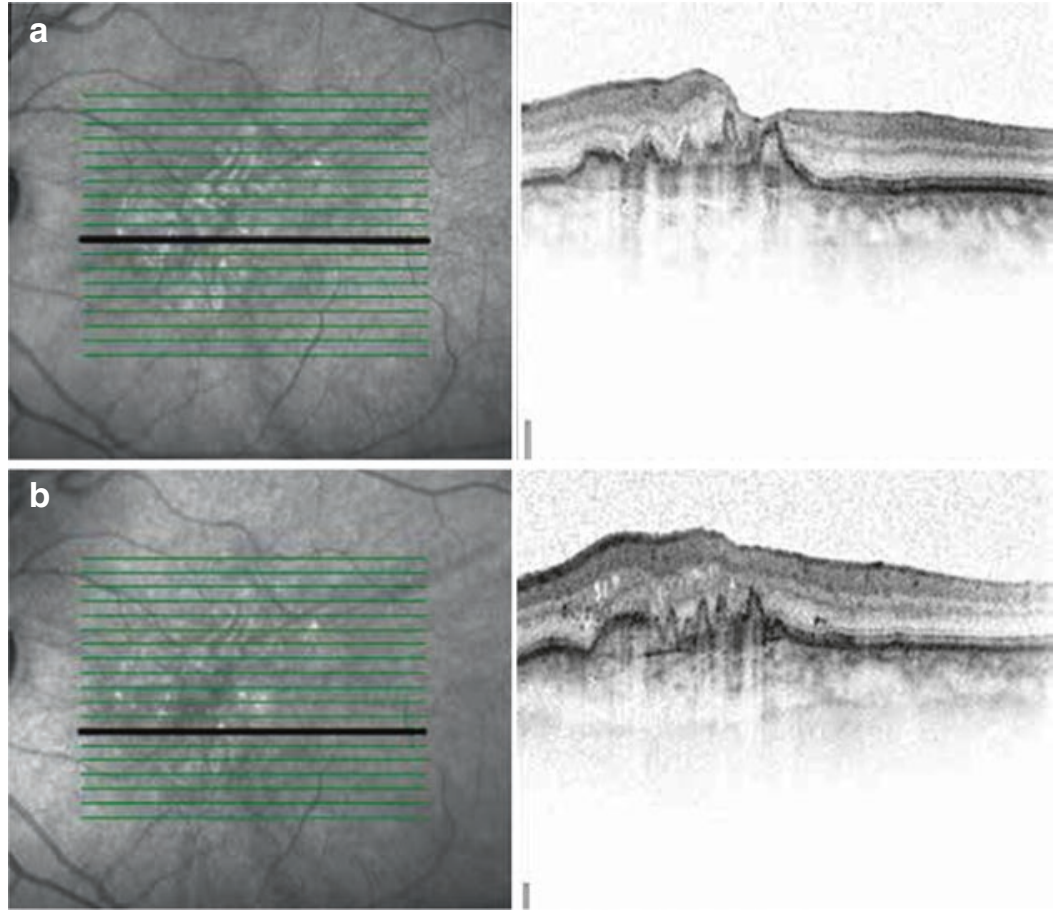
challenging with an increasing number of B-scans. In addition, the exact assignment of OCT layers to anatomical structures (applied after the consensus of the International Nomenclature for Optical Coherence Tomography Panel) as well as pattern recognition plays an important role in image evaluation, as the differential diagnosis or treatment indication can be supported by recognition of characteristic OCT findings. For example, domeshaped elevations of the RPE in the presence of soft drusen indicate exudative AMD; whereas marked thickening of the choroid in OCT images without soft drusen would point to CSCR. Further examples of challenging OCT interpretations are demonstrated in Fig. 4.14.
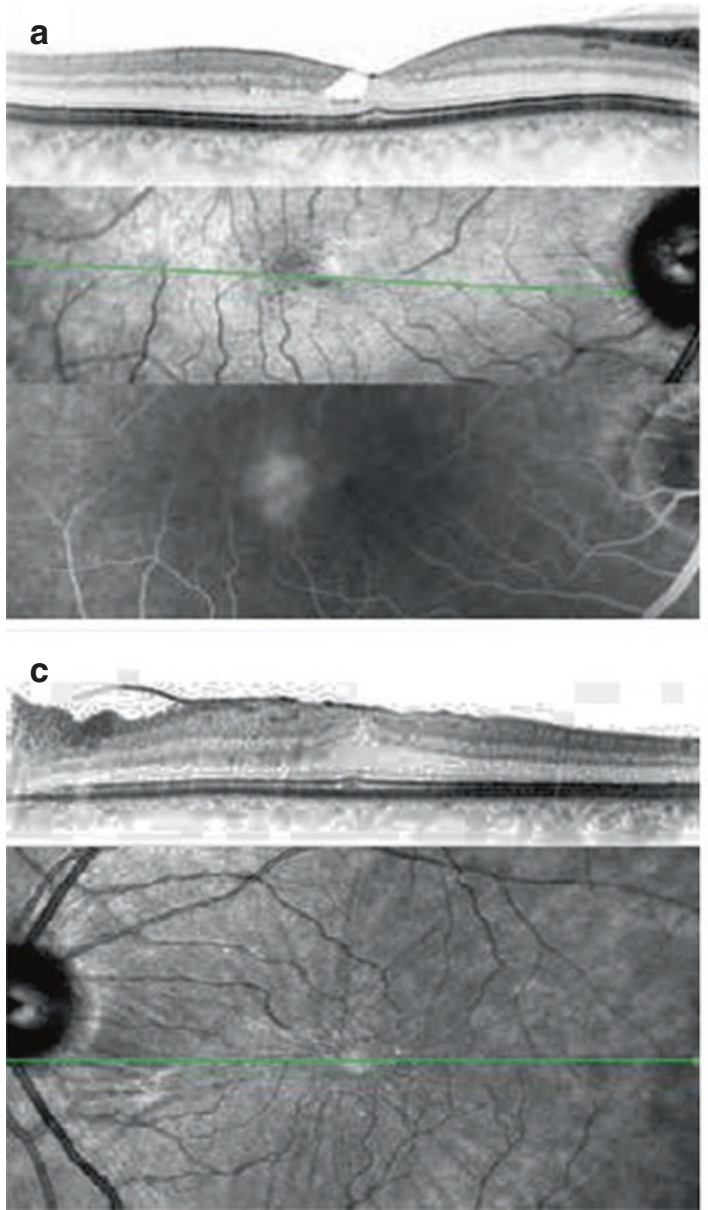

Fig. 4.14 Pitfalls in OCT interpretation II. In contrast to intraretinal cystoid lesions secondary to $\mathrm{CNV}$, macular telangiectasia type 2 revealed mimicking alterations within the inner retina, due to degenerative changes (a). Note that there is no thickening of the retina. Further misinterpretation concerning indication to anti-VEGF treatment might derive from
Projection artefacts may derive from hyperreflective changes in the vitreous (e.g., floaters) or on the surface of the retina (e.g., epiretinal membranes) that may lead to suppression of structures in deeper retinal layers. In such scenarios, comparison with other imaging modalities or ophthalmoscopy is helpful. When applying automatic analysis algorithms, operators and clinicians should evaluate the segmentation of retinal boundaries in each B-scan and, if necessary, manually correct them [61].

The quantitative evaluation of OCT findings requires precise definition of individual parameters. To date, there is no industry standard or consensus, with different terms being used in

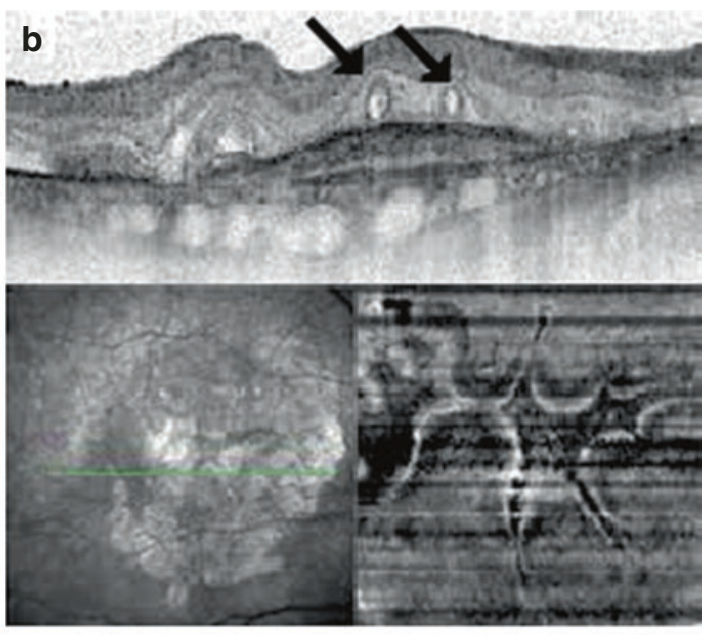

d

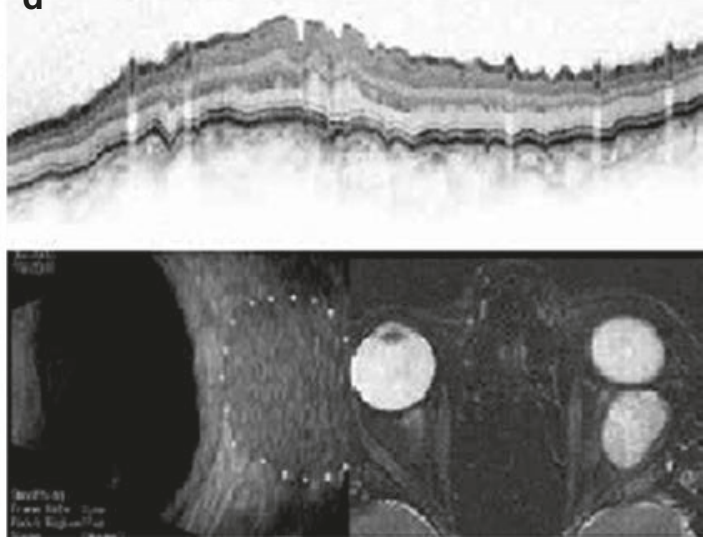

degenerative outer retinal tubulations (arrows) as visualized by an OCT B-scan and by an OCT en face image (b), epiretinal membrane (c), or choroidal folds of different origins (e.g. orbital tumor, (d)). Source: Schmitz-Valckenberg S et al.: Pitfalls in retinal OCT imaging. Ophthalmology @ Point of Care. 2017. Reprinted with permission 
parallel. The correct geometric location and segmentation of relevant anatomic landmarks is crucial for meaningful and correct quantitative analysis. But the definition of landmarks such as the fovea may be challenging in the presence of pathologic changes. The OCT interpretation is usually based on the 1:1 pixel presentation mode, in which the image information in the lateral compared to the anteroposterior dimension is compressed depending on the device. It was shown that the quantification of areas or distances in the 1:1 pixel presentation mode is prone to overestimation of values in the anteroposterior dimension. Therefore, measurements should be performed in the $1: 1 \mu \mathrm{m}$ presentation mode [64, 65]. Inaccuracies of measurements within B-scans may further occur if the retinal layers are not orthogonal to the laser beam. To determine correct values, measurements should always be performed parallel to the beam path. Furthermore, the method of scaling must be considered when measured values are specified in the metric system.

Several structures are usually not distinguishable, because of the lack of reflectivity in the perpendicular laser beam. Changing the direction of the laser beam in relation to the retina, e.g. by tilting the head of the subjects, some of these structures, like the Henle fiber layer, may become visible [66]. However, other alterations, like retinal hemorrhages, stay concealed as the imaging light shows little or no interference in the area of bleeding (Fig. 4.15).

In conclusion, while OCT alone should not be the only basis for diagnostic and treatment recommendations, its application in daily clinical practice and for research purposes has become invaluable. It should be regarded as an additional diagnostic procedure in a multimodal imaging assessment including fundoscopy, fluorescence angiography, in association with a careful anamnesis and assessment of patient's complaints. The latter is known to differ frequently from the severity of OCT findings. In these cases, it is more than mandatory to perform complimentary imaging and diagnostic procedures [61].
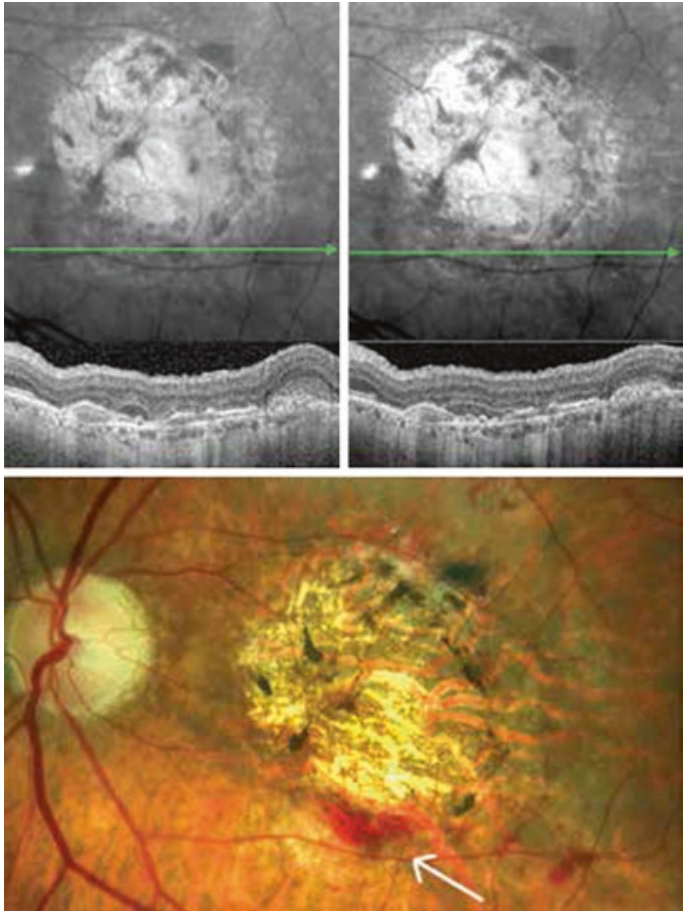

Fig. 4.15 Pitfalls in OCT interpretation III. Hemorrhages (arrow on fundus photograph) are frequently not visualized by near-infrared reflection and optical coherence tomography imaging due to the wavelength used. Source: Schmitz-Valckenberg $S$ et al.: Pitfalls in retinal OCT imaging. Ophthalmology @ Point of Care. 2017. Reprinted with permission

\subsection{Summary and Outlook}

Many posterior segment ocular diseases involve both the retina and the choroid as the RPE, Bruch's membrane and the choroid represent a coadjutant functional complex [67]. This may be particularly important in retinal disorders such as AMD, the most common cause of legal blindness in industrialized countries, characterized by abnormal extracellular material deposition either below or above the retinal pigment epithelial layer [68, 69]. Even single gene retinal dystrophies like $A B C A 4$-related retinopathy, that primarily affects the RPE by excessive accumulation of lipofuscin, or pseudoxanthoma elasticum (PXE), which leads to a calcification of the Bruch's membrane, have been described to reveal 
choroidal alterations $[54,55,70,71]$. The combination of shorter and longer wavelength light sources within one gadget might combine the advantages attributed to SD-OCT (i.e., better resolution for the visualization of retinal layers) and SS-OCT (i.e., visualization of the choroid). This might allow for optimum visualization of intraretinal as well as subretinal structures without temporal or spatial separation. In 2017, the first OCT device using different wavelengths of laser light sources was built at the Technical University of Biel and University of Basel, Switzerland. First clinical data and value with the device remain to be demonstrated as well as the possible commercial feasibility.

Since the beginning, continuous improvements have been made to scan rates as well as axial and lateral resolution. Commercial OCT systems achieve scan rates up to $250,000 \mathrm{~Hz}$ and an axial resolution of under $7 \mu \mathrm{m}[1,6]$. Faster imaging improves patient comfort and reduces acquisition time, increasing the likelihood of better scan quality. It also enables volumetric as well as 3-dimensional analysis of various pathological features, including choroidal neovascularization and intraretinal fluid. The latter might help in monitoring disease progression and treatment effects [72]. Furthermore, higher quality by improved resolution will further enhance automated segmentation and analysis, a field of rising importance in the view of growing applications of artificial intelligence and machine learning in ophthalmology [73-75].

During the last decades, OCT technology has revolutionized the retina subspecialty field. OCT imaging now plays a pivotal role in understanding, diagnosing, and monitoring natural history and treatment effects in AMD, diabetic retinopathy, retinal vascular diseases, CSCR, high myopia and many other retinal and choroidal conditions. High resolution and high-quality multimodal assessment in combination with continuous innovations of the OCT imaging modality are aiming to further improve the clinical assessment of retinal and choroidal diseases.

\section{References}

1. Litts KM, Zhang Y, Freund KB, et al. Optical coherence tomography and histology of age-rrelated macular degeneration support mitochondria as reflectivity sources. Retina. 2018;38:445-61. https://doi. org/10.1097/IAE.0000000000001946.

2. Adhi M, Duker JS. Optical coherence tomography--current and future applications. Curr Opin Ophthalmol. 2013;24:213-21. https://doi. org/10.1097/ICU.0b013e32835f8bf8.

3. Hee MR, Izatt JA, Swanson EA, et al. Optical coherence tomography of the human retina. Arch Ophthalmol. 1995;113:325-32. http://www.ncbi.nlm. nih.gov/pubmed/7887846. Accessed 24 May 2018.

4. Puliafito CA, Hee MR, Lin CP, et al. Imaging of macular diseases with optical coherence tomography. Ophthalmology. 1995;102:217-29. http://www.ncbi. nlm.nih.gov/pubmed/7862410. Accessed 24 May 2018.

5. Choma M, Sarunic M, Yang C, et al. Sensitivity advantage of swept source and Fourier domain optical coherence tomography. Opt Express. 2003;11:2183. https://doi.org/10.1364/OE.11.002183.

6. Cereda MG, Corvi F, Cozzi M, et al. Optical coherence tomography 2. Retina. 2019;39:415. https://doi. org/10.1097/IAE.0000000000001953.

7. Corvi F, Pellegrini M, Erba S, et al. Reproducibility of vessel density, fractal dimension, and foveal avascular zone using 7 different optical coherence tomography angiography devices. Am J Ophthalmol. 2018;186:2531. https://doi.org/10.1016/j.ajo.2017.11.011.

8. Menke MN, Dabov S, Knecht P, et al. Reproducibility of retinal thickness measurements in healthy subjects using spectralis optical coherence tomography. Am J Ophthalmol. 2009;147:467-72. https://doi. org/10.1016/j.ajo.2008.09.005.

9. Margolis R, Spaide RF. A pilot study of enhanced depth imaging optical coherence tomography of the choroid in normal eyes. Am J Ophthalmol. 2009;147:811-5. https://doi.org/10.1016/j.ajo.2008.12.008.

10. Unterhuber A, Povazay B, Hermann B, et al. In vivo retinal optical coherence tomography at $1040 \mathrm{~nm}$ - enhanced penetration into the choroid. Opt Express. 2005;13:3252. https://doi.org/10.1364/ OPEX.13.003252.

11. Povazay B, Bizheva K, Hermann B, et al. Enhanced visualization of choroidal vessels using ultrahigh resolution ophthalmic OCT at $1050 \mathrm{~nm}$. Opt Express. 2003;11:1980. https://doi.org/10.1364/ OE.11.001980

12. An L, Li P, Lan G, et al. High-resolution 1050 nm spectral domain retinal optical coherence tomography at $120 \mathrm{kHz} \mathrm{A}$-scan rate with $61 \mathrm{~mm}$ imaging depth. Biomed Opt Express. 2013;4:245. https://doi. org/10.1364/BOE.4.000245. 
13. Puvanathasan P, Forbes P, Ren Z, et al. High-speed, high-resolution Fourier-domain optical coherence tomography system for retinal imaging in the $1060 \mathrm{~nm}$ wavelength region. Opt Lett. 2008;33:2479. https://doi.org/10.1364/OL.33.002479.

14. Klein R, Klein BE, Linton KL. Prevalence of agerelated maculopathy. The Beaver Dam eye study. Ophthalmology. 1992;99:933-43. http://www.ncbi. nlm.nih.gov/pubmed/1630784. Accessed 5 Dec 2018.

15. Jager RD, Mieler WF, Miller JW. Age-related macular degeneration. N Engl J Med. 2008;358:2606-17. https://doi.org/10.1056/NEJMra0801537.

16. Ferris FL, Wilkinson CP, Bird A, et al. Clinical classification of age-related macular degeneration. Ophthalmology. 2013;120:844-51. https://doi. org/10.1016/j.ophtha.2012.10.036.

17. Khanifar AA, Koreishi AF, Izatt JA, et al. Drusen ultrastructure imaging with spectral domain optical coherence tomography in age-related macular degeneration. Ophthalmology. 2008;115:1883-90. https:// doi.org/10.1016/j.ophtha.2008.04.041.

18. Gorczynska I, Srinivasan VJ, Vuong LN, et al. Projection OCT fundus imaging for visualising outer retinal pathology in non-exudative age-related macular degeneration. Br J Ophthalmol. 2009;93:603-9. https://doi.org/10.1136/bjo.2007.136101.

19. Sikorski BL, Bukowska D, Kaluzny JJ, et al. Drusen with accompanying fluid underneath the sensory retina. Ophthalmology. 2011;118:82-92. https://doi. org/10.1016/j.ophtha.2010.04.017.

20. Schuman SG, Koreishi AF, Farsiu S, et al. Photoreceptor layer thinning over drusen in eyes with age-related macular degeneration imaged in vivo with spectral-domain optical coherence tomography. Ophthalmology. 2009;116:488-496.e2. https://doi. org/10.1016/j.ophtha.2008.10.006.

21. Gass JD. A clinicopathologic study of a peculiar foveomacular dystrophy. Trans Am Ophthalmol Soc. 1974;72:139-56. http://www.ncbi.nlm.nih.gov/ pubmed/4142662. Accessed 5 Dec 2018.

22. Gass JD, Jallow S, Davis B. Adult vitelliform macular detachment occurring in patients with basal laminar drusen. Am J Ophthalmol. 1985;99:445-59. http:// www.ncbi.nlm.nih.gov/pubmed/3985082. Accessed 5 Dec 2018.

23. Russell SR, Mullins RF, Schneider BL, et al. Location, substructure, and composition of basal laminar drusen compared with drusen associated with aging and age-related macular degeneration. Am J Ophthalmol. 2000;129:205-14. http://www.ncbi.nlm.nih.gov/ pubmed/10682974. Accessed 5 Dec 2018.

24. Finger RP, Charbel Issa P, Kellner U, et al. Spectral domain optical coherence tomography in adult-onset vitelliform macular dystrophy with cuticular drusen. Retina. 2010;30:1455-64. https://doi.org/10.1097/ IAE.0b013e3181e09829.

25. Klein R, Meuer SM, Knudtson MD, et al. The epidemiology of retinal reticular drusen. Am J Ophthalmol. 2008;145:317-26. https://doi. org/10.1016/j.ajo.2007.09.008.
26. Mimoun G, Soubrane G, Coscas G. [Macular drusen]. J Fr Ophtalmol 1990;13:511-530. http://www.ncbi. nlm.nih.gov/pubmed/2081842. Accessed 5 Dec 2018.

27. Keane PA, Patel PJ, Liakopoulos S, et al. Evaluation of age-related macular degeneration with optical coherence tomography. Surv Ophthalmol. 2012;57:389-414. survophthal.2012.01.006.

28. Freund KB, Laud K, Lima LH, et al. Acquired Vitelliform Lesions: correlation of clinical findings and multiple imaging analyses. Retina. 2011;31:1325. https://doi.org/10.1097/IAE.0b013e3181ea48ba.

29. Holz FG, Pauleikhoff D, Klein R, et al. Pathogenesis of lesions in late age-related macular disease. Am J Ophthalmol. 2004;137:504-10. https://doi. org/10.1016/j.ajo.2003.11.026.

30. Wolf-Schnurrbusch UEK, Enzmann V, Brinkmann $\mathrm{CK}$, et al. Morphologic changes in patients with geographic atrophy assessed with a novel spectral OCT-SLO combination. Investig Opthalmol Vis Sci. 2008;49:3095. https://doi.org/10.1167/iovs.07-1460.

31. Fleckenstein M, Schmitz-Valckenberg S, Adrion $\mathrm{C}$, et al. Tracking progression with spectral-domain optical coherence tomography in geographic atrophy caused by age-related macular degeneration. Invest Ophthalmol Vis Sci. 2010;51:3846-52. https://doi. org/10.1167/iovs.09-4533.

32. Schmitz-Valckenberg S, Fleckenstein M, Helb H-M, et al. In vivo imaging of foveal sparing in geographic atrophy secondary to age-related macular degeneration. Investig Opthalmol Vis Sci. 2009;50:3915. https://doi.org/10.1167/iovs.08-2484.

33. Fleckenstein M, Schmitz-Valckenberg S, Martens C, et al. Fundus autofluorescence and spectral-domain optical coherence tomography characteristics in a rapidly progressing form of geographic atrophy. Invest Ophthalmol Vis Sci. 2011;52:3761-6. https://doi. org/10.1167/iovs.10-7021.

34. Manjunath V, Goren J, Fujimoto JG, et al. Analysis of choroidal thickness in age-related macular degeneration using spectral-domain optical coherence tomography. Am J Ophthalmol. 2011;152:663-8. https:// doi.org/10.1016/j.ajo.2011.03.008.

35. Spaide RF. Age-related choroidal atrophy. Am J Ophthalmol. 2009;147:801-10. https://doi. org/10.1016/j.ajo.2008.12.010.

36. Grossniklaus HE, Green WR. Choroidal neovascularization. Am J Ophthalmol. 2004;137:496-503. https://doi.org/10.1016/j.ajo.2003.09.042.

37. Yannuzzi LA, Negrão S, Iida T, et al. Retinal angiomatous proliferation in age-related macular degeneration. Retina. 2012;32(Suppl 1):416-34. http://www. ncbi.nlm.nih.gov/pubmed/22451953. Accessed 6 Dec 2018.

38. Green WR. Histopathology of age-related macular degeneration. Mol Vis. 1999;5:27. http://www.ncbi. nlm.nih.gov/pubmed/10562651. Accessed 6 Dec 2018.

39. Rosenfeld PJ. Optical coherence tomography and the development of antiangiogenic therapies in neo- 
vascular age-related macular degeneration. Investig Opthalmol Vis Sci. 2016;57:OCT14. https://doi. org/10.1167/iovs.16-19969.

40. Kim S-W, Oh J, Kwon S-S, et al. Comparison of choroidal thickness among patients with healthy eyes, early age-related maculopathy, neovascular agerelated macular degeneration, central serous chorioretinopathy, and polypoidal choroidal vasculopathy. Retina. 2011;31:1904-11. https://doi.org/10.1097/ IAE.0b013e31821801c5.

41. Bartsch DU, Weinreb RN, Zinser G, et al. Confocal scanning infrared laser ophthalmoscopy for indocyanine green angiography. Am J Ophthalmol. 1995;120:642-51. http://www.ncbi.nlm.nih.gov/ pubmed/7485366. Accessed 6 Dec 2018.

42. Shiragami C, Shiraga F, Matsuo T, et al. Risk factors for diabetic choroidopathy in patients with diabetic retinopathy. Graefes Arch Clin Exp Ophthalmol. 2002;240:43642. https://doi.org/10.1007/s00417-002-0451-5.

43. Bin WW, Xu L, Jonas JB, et al. Subfoveal choroidal thickness: the Beijing Eye Study. Ophthalmology. 2013;120:175-80. https://doi.org/10.1016/j. ophtha.2012.07.048.

44. Elman MJ, Aiello LP, Beck RW, et al. Randomized trial evaluating ranibizumab plus prompt or deferred laser or triamcinolone plus prompt laser for diabetic macular edema. Ophthalmology. 2010;117:1064-1077.e35. https://doi.org/10.1016/j. ophtha.2010.02.031.

45. Virgili G, Menchini F, Dimastrogiovanni AF, et al. Optical coherence tomography versus stereoscopic fundus photography or biomicroscopy for diagnosing diabetic macular edema: a systematic review. Investig Opthalmol Vis Sci. 2007;48:4963. https://doi. org/10.1167/iovs.06-1472.

46. Romero-Aroca P. Current status in diabetic macular edema treatments. World J Diabetes. 2013;4:165. https://doi.org/10.4239/wjd.v4.i5.165.

47. Esmaeelpour M, Považay B, Hermann B, et al. Mapping choroidal and retinal thickness variation in type 2 diabetes using three-dimensional 1060-nm optical coherence tomography. Invest Ophthalmol Vis Sci. 2011;52:5311-6. https://doi.org/10.1167/ iovs.10-6875.

48. Raafay S, Campochiaro PA. Treatment of macular edema following branch retinal vein occlusion. US Ophthalmic Rev. 2013;06:148. https://doi. org/10.17925/USOR.2013.06.02.148.

49. Gemenetzi M, De Salvo G, Lotery AJ. Central serous chorioretinopathy: an update on pathogenesis and treatment. Eye. 2010;24:1743-56. https://doi. org/10.1038/eye.2010.130.

50. Pryds A, Larsen M. Choroidal thickness following extrafoveal photodynamic treatment with verteporfin in patients with central serous chorioretinopathy. Acta Ophthalmol. 2012;90:738-43. https://doi. org/10.1111/j.1755-3768.2011.02157.x.

51. Adatia FA, Luong M, Munro M, et al. The other CNVM: a review of myopic choroidal neovascularization treatment in the age of anti-vascular endothelial growth factor agents. Surv Ophthalmol. 2015;60:20415. https://doi.org/10.1016/j.survophthal.2014.10.002.

52. Lorenzo D, Arias L, Choudhry N, et al. Dome-shaped macula in myopic eyes. Retina. 2017;37:680-6. https://doi.org/10.1097/IAE.0000000000001222.

53. Charbel Issa P, Finger RP, Kruse K, et al. Monthly ranibizumab for nonproliferative macular telangiectasia type 2: a 12-month prospective study. Am J Ophthalmol. 2011;151:876-886.e1. https://doi. org/10.1016/j.ajo.2010.11.019.

54. Müller PL, Fimmers R, Gliem M, et al. Choroidal alterations in ABCA4-related retinopathy. Retina. 2017;37:359-67. https://doi.org/10.1097/ IAE.0000000000001169.

55. Gliem M, Fimmers R, Müller PL, et al. Choroidal changes associated with Bruch membrane pathology in pseudoxanthoma elasticum. Am J Ophthalmol. 2014;158:198-207.e3. https://doi.org/10.1016/j. ajo.2014.04.005.

56. Dhoot DS, Huo S, Yuan A, et al. Evaluation of choroidal thickness in retinitis pigmentosa using enhanced depth imaging optical coherence tomography. $\mathrm{Br} \mathrm{J}$ Ophthalmol. 2013;97:66-9. https://doi.org/10.1136/ bjophthalmol-2012-301917.

57. Freton A, Finger PT. Spectral domain-optical coherence tomography analysis of choroidal osteoma. $\mathrm{Br} \mathrm{J}$ Ophthalmol. 2012;96:224-8. https://doi.org/10.1136/ bjo.2011.202408.

58. Torres VLL, Brugnoni N, Kaiser PK, et al. Optical coherence tomography enhanced depth imaging of choroidal tumors. Am J Ophthalmol. 2011;151:586593.e2. https://doi.org/10.1016/j.ajo.2010.09.028.

59. Fardeau C, Champion E, Massamba N, et al. Uveitic macular edema. Eye. 2016;30:1277-92. https://doi. org/10.1038/eye.2016.115.

60. Duker JS, Kaiser PK, Binder S, et al. The International Vitreomacular Traction Study Group classification of vitreomacular adhesion, traction, and macular hole. Ophthalmology. 2013;120:2611-9. https://doi. org/10.1016/j.ophtha.2013.07.042.

61. Schmitz-Valckenberg S, Brinkmann CK, Heimes B, et al. Pitfalls in retinal OCT imaging. Ophthalmologe. 2017;1:oapoc.0000024. https://doi. org/10.5301/oapoc.0000024.

62. Rahimy E, Rayess N, Maguire JI, et al. Radial versus raster spectral-domain optical coherence tomography scan patterns for detection of macular pathology. Am J Ophthalmol. 2014;158:345-353.e2. https://doi. org/10.1016/j.ajo.2014.05.013.

63. Hwang YH, Lee JY, Kim YY. The effect of head tilt on the measurements of retinal nerve fibre layer and macular thickness by spectral-domain optical coherence tomography. Br J Ophthalmol. 2011;95:154751. https://doi.org/10.1136/bjo.2010.194118.

64. Tan CSH, Cheong KX, Sadda SR. Overestimation of subfoveal choroidal thickness by measurement based on horizontally compressed optical coherence tomography images. Graefes Arch Clin Exp Ophthalmol. 2013;251:2835-6. https://doi.org/10.1007/ s00417-013-2454-9. 
65. Kim JH, Kang SW, Ha HS, et al. Overestimation of subfoveal choroidal thickness by measurement based on horizontally compressed optical coherence tomography images. Graefes Arch Clin Exp Ophthalmol. 2013;251:1091-6. https://doi.org/10.1007/ s00417-012-2147-9.

66. Lujan BJ, Roorda A, Knighton RW, et al. Revealing Henle's fiber layer using spectral domain optical coherence tomography. Invest Ophthalmol Vis Sci. 2011;52:1486-92. https://doi.org/10.1167/ iovs.10-5946.

67. Saint-Geniez M, Kurihara T, Sekiyama E, et al. An essential role for RPE-derived soluble VEGF in the maintenance of the choriocapillaris. Proc Natl Acad Sci U S A. 2009;106:18751-6. https://doi. org/10.1073/pnas.0905010106.

68. Holz FG, Sadda SR, Staurenghi G, et al. Imaging protocols in clinical studies in advanced agerelated macular degeneration: recommendations from classification of atrophy consensus meetings. Ophthalmology. 2017;124:464-78. https:// doi.org/10.1016/j.ophtha.2016.12.002.

69. Spaide RF. Choriocapillaris flow features follow a power law distribution: implications for characterization and mechanisms of disease progression.
Am J Ophthalmol. 2016;170:58-67. https://doi. org/10.1016/j.ajo.2016.07.023.

70. Pellegrini M, Acquistapace A, Oldani M, et al. Dark atrophy: an optical coherence tomography angiography study. Ophthalmology. 2016;123:1879-86. https://doi.org/10.1016/j.ophtha.2016.05.041.

71. Giani A, Pellegrini M, Carini E, et al. The dark atrophy with indocyanine green angiography in stargardt disease. Investig Opthalmol Vis Sci. 2012;53:3999. https://doi.org/10.1167/iovs.11-9258.

72. Drexler W, Fujimoto JG. State-of-the-art retinal optical coherence tomography. Prog Retin Eye Res. 2008;27:45-88. https://doi.org/10.1016/j. preteyeres.2007.07.005.

73. Lee CS, Lee AY, Holland GN, et al. Big data and uveitis. Ophthalmology. 2016;123:2273-5. https://doi. org/10.1016/j.ophtha.2016.08.037.

74. Lee A, Taylor P, Kalpathy-Cramer J, et al. Machine learning has arrived! Ophthalmology. 2017;124:17268. https://doi.org/10.1016/j.ophtha.2017.08.046.

75. Tufail A, Rudisill C, Egan C, et al. Automated diabetic retinopathy image assessment software. Ophthalmology. 2017;124:343-51. https://doi. org/10.1016/j.ophtha.2016.11.014.

Open Access This chapter is licensed under the terms of the Creative Commons Attribution 4.0 International License (http://creativecommons.org/licenses/by/4.0/), which permits use, sharing, adaptation, distribution and reproduction in any medium or format, as long as you give appropriate credit to the original author(s) and the source, provide a link to the Creative Commons license and indicate if changes were made.

The images or other third party material in this chapter are included in the chapter's Creative Commons license, unless indicated otherwise in a credit line to the material. If material is not included in the chapter's Creative Commons license and your intended use is not permitted by statutory regulation or exceeds the permitted use, you will need to obtain permission directly from the copyright holder. 